\title{
Reply to the Editor: "Is There a Role of Surgery in Treating Localized Esophageal Neuroendocrine Tumor?"
}

\author{
Suna Erdem, MD ${ }^{1}$, Rene Warschkow, $\mathrm{MD}^{2}$, and Mathias Worni, MD, MHS \\ ${ }^{1}$ Moores Cancer Center, University of California San Diego (UCSD), San Diego, CA; ${ }^{2}$ Department of Surgery, \\ Kantonsspital, St. Gallen, Switzerland; ${ }^{3}$ Clarunis, Department of Visceral Surgery, University Centre for Gastrointestinal \\ and Liver Diseases, St. Clara Hospital and University Hospital, Basel, Switzerland
}

\section{Dear Editor,}

We greatly appreciate the interest of Dr. Deng in our article, "Is there a role for surgery in patients with neuroendocrine tumors of the esophagus? - a contemporary view from the NCDB," which was recently published in Annals of Surgical Oncology. ${ }^{1}$ We appreciate the opportunity to respond to his comments about whether all patients with localized, esophageal neuroendocrine tumor (eNET) benefit from surgical therapy.

In our study, we analyzed overall survival (OS) of histologically confirmed stage I-III, primary eNETs according to their treatment modalities. In general, we were able to demonstrate that patients who were treated with surgical resection of the primary tumor had an overall survival benefit compared with patients who did not undergo surgical resection. This survival benefit held true even after application of advanced statistical modeling.

However, we agree with Dr. Deng that surgical resection of patients with eNETs is not recommended for all patients with localized tumors. Based on data from NCDB, many subgroups of patients were less likely to undergo surgical resection compared with their counterparts. This emphasizes that during the time period of the study, patients were already differently selected to undergo surgery. One of the strong predictors was tumor stage. While there was no difference between stage I and II disease, patients with stage III disease were less likely to undergo surgery. Based on subgroup analyses focusing on stage I and II disease, overall survival was increased for those with resection. This also holds true

(C) Society of Surgical Oncology 2020

First Received: 26 November 2019;

Published Online: 11 December 2019

S. Erdem, MD

e-mail: serdem@ucsd.edu

M. Worni, MD, MHS

e-mail: mathias.worni@duke.edu by limiting patients to stage III disease with a univariate hazard ratio (HR) for resection of 0.59 (95\% confidence interval [CI] $0.36-0.98, p=0.003)$. This result was confirmed after applying propensity score methods with an HR of 0.38 (95\% CI $0.16-0.90, p=0.015)$. These results show that our findings differ to some extent from the findings by Deng et al. as even patients with stage III disease seem to benefit from surgical resection. ${ }^{2}$

Despite analyzing large datasets and applying latest statistical methods, this difference in retrospective data highlights the need for prospective studies if possible. Until then, the statement that patients with stage III disease should not undergo surgery is controversial and will need further investigations. For now, patients with stage III disease should at least be evaluated for surgical resection, which should be done in perspective of the multiple other prognostic factors, such as tumor grade. Given that eNET is such a rare disease, further stratification into subgroups is not possible for statistical reasons, and physicians are confined to imperfect data. As such, we agree that the recommendations for surgical resection among patients with stage I/II disease is stronger. Stage III disease should not per se preclude surgical resection, but the selection should be much more rigorous.

DISCLOSURES The authors declare no conflicts of interest.

\section{REFERENCES}

1. Erdem $\mathrm{S}$, et al. Is there a role for surgery in patients with neuroendocrine tumors of the esophagus? A contemporary view from the NCDB. Ann Surg Oncol. 2019. https://doi.org/10.1245/ s10434-019-07847-1.

2. Deng HY, et al. Neuroendocrine carcinoma of the esophagus: clinical characteristics and prognostic evaluation of 49 cases with surgical resection. J Thorac Dis. 2016;8(6):1250-6.

Publisher's Note Springer Nature remains neutral with regard to jurisdictional claims in published maps and institutional affiliations. 